Gut, 1960, 1, 303.

\title{
SERUM ALBUMIN, PSEUDOCHOLINESTERASE, AND TRANSAMINASES IN THE ASSESSMENT OF LIVER FUNCTION BEFORE AND AFTER VENOUS SHUNT OPERATIONS*
}

\author{
BY \\ ALAN H. HUNT and HERMANN LEHMANN
}

From St. Bartholomew's Hospital, London

This paper records biochemical and follow-up observations on 153 patients with cirrhosis who have had a shunt operation. The experience confirms that no shunt operation should be undertaken on a patient whose serum albumin level is less than $3.2 \mathrm{~g} . \%$. Low pseudocholinesterase levels are of help diagnostically but the main value of this test is in indicating whether or not liver function will deteriorate after a shunt operation in any given patient.

The selection of cirrhotic patients for porta-caval anastomosis is a complex matter, since only a proportion of those for whom operation is indicated are suitable. The indications for operation are clinical, in that the operations are designed to prevent or to stop haemorrhage from gastrooesophageal varices, to reduce or to neutralize the anaemia, etc., of an overactive spleen, and, occasionally, to relieve ascites. The means of achieving the desired effect on the portal circulation is to divert portal blood directly into the systemic circulation and, of necessity, away from the liver. It is, therefore, essential for the patients to have a certain minimum reserve of liver function so that they will not die of liver failure during the immediate post-operative phase. It is then to be hoped that the liver function will be maintained over the years that follow so that the patients will be able to lead normal lives and continue at work.

The purpose of this communication is to relate the findings of certain tests of liver function to the clinical results obtained in a group of patients operated on before October, 1959, and to evaluate them as a means of assessing the prognosis of a patient about to undergo a shunt operation. In this context, diagnostic tests of liver disease are no more than adjuvant, as indeed are tests of biliary obstruction and the capacity of the liver to eliminate bromsulphthaleine and to form prothrombin. We do not wish to minimize in any way the importance of these tests and other means of estimating how

- Based on a paper read to the joint meeting of the Association of Clinical Pathologists and the Association of Clinical Biochemists on October 3, 1959. much of a specific liver function remains or the extent of its derangement. We realized early in our studies that the tests which would serve us best in the assessment of prognosis would be those which would give a direct estimation of the function of the liver as reflected by its capacity to manufacture substances vital to the metabolism of the organism as a whole. Two serum components, albumin and pseudocholinesterase, are just such substances. They are made by the liver and their concentration indicates how much normal liver function is present. Malnutrition, haemodilution, and variations in the normal values must be allowed for in the evaluation.

We also realized that many patients died because their liver disease continued to progress. At no time has it been pretended that a surgical procedure could arrest such a degenerative process. Recently we have been investigating the serum glutamicoxalacetic and glutamic-pyruvic transaminases, in the hope that they might show how much active disease was present within the liver at the time a shunt operation was done and possibly indicate those patients in whom deterioration might be expected, they having survived the porta-caval anastomosis.

\section{Serum Albumin}

Up to the end of 1954 albumin was estimated by the biuret method after precipitation of the globulins by half-saturation with ammonium sulphate (Harrison, 1937). From 1955, globulin precipitation after Kingsley's method with ether and sodium sulphate was used (Hawk, Oser, and Summerson, 1947). The lower level of normal was considered 
to be $3.5 \mathrm{~g}$. per $100 \mathrm{ml}$. of serum. Differences in results of duplicate estimations or of estimations done on the same serum on different days did not exceed $0 \cdot 1 \mathrm{~g}$. albumin per $100 \mathrm{ml}$. serum.

The level of serum albumin has for some time been recognized to be of prognostic value. Blakemore as long ago as 1949 was emphatic in his opinion that shunt operations should not be undertaken on patients with serum albumin levels of less than 3.2 g. per $100 \mathrm{ml}$. We do not attach undue importance to a single laboratory figure to the exclusion of more empirical and less easily defined clinical methods of assessment, but we agree in general with Blakemore's opinion, provided the estimation is carried out reliably, recognizing that standards vary from one laboratory to another. This one test, above all others, helps in the selection of patients likely to survive the shunt operation itself. After that, some patients do well, some deteriorate and ultimately die, and in some the clinical course fluctuates. The pre-operative serum albumin level gives disappointingly little indication of those patients in whom the liver will ultimately fail.

\section{Pseudocholinesterase}

Our attention was drawn to this test in 1952 by the late Professor Bruno Mendel, of the University of Amsterdam, who suggested that it might prove valuable as a prognostic test complementary to the serum albumin. The method of estimation has throughout been that of Jones and Tod (1935) and McArdle (1940). It depends on the measurement in a Warburg micromanometer of the amount of $\mathrm{CO}_{2}$ liberated when acetylcholine is added to a mixture of serum and Krebs-Ringer bicarbonate buffer.

The esterase hydrolyses acetylcholine into choline and acetic acid, and the acid liberates $\mathrm{CO}_{2}$ from bicarbonate. Results are expressed in c.mm. (units) of $\mathrm{CO}_{2}$ liberated by $1 \mathrm{ml}$. of serum in one minute at $37^{\circ} \mathrm{C}$. Measurements are made in duplicate and do not differ by more than 6 units. The mean of the two results is recorded.

In a series of 100 adults without disease values varied from 65 to 124 units. For practical purposes only values under 55 units were considered abnormal. We have come to realize, while making due allowance for the wide range that exists between individuals, that there is a critical level of 30 to 35 units. The interpretation of single measurements has been complicated by the finding of a rare genetically determined pseudocholinesterase deficiency which is unrelated to liver disease or malnutrition. All homozygotes and a proportion of the heteroyzgotes for the abnormal gene show values below 55 units (Lehmann and Ryan, 1956; Lehmann, Patston, and Ryan, 1958). Kalow and his colleagues (Kalow and Genest, 1957; Kalow and Gunn, 1959) have shown that such low pseudocholinesterase values are associated with an atypical enzyme which has a low affinity for acetylocholine. Harris, Whittaker, Lehmann, and Silk (1960) have examined 58 immediate relatives of 11 homozygotes for the abnormal gene and have confirmed that homo- and heterozygotes can be recognized from tests demonstrating the atypical pseudocholinesterase. It is, therefore, possible to show whether a low pseudocholinesterase level is due to a fall in the concentration of the normal enzyme or due to full or partial replacement by the genetically determined atypical esterase.

\section{Serum Transaminases}

The glutamic-oxalacetic and the glutamic-pyruvic transaminases were determined by the method of King (1958) and Reitman and Frankel (1957) using a routine photoelectric colorimeter with a green filter (Lehmann and Ryan, 1957). Whereas serum albumin and serum pseudocholinesterase are made by the liver and their concentration is a direct outcome of liver function, concentrations of serum transaminases are raised when liver cells are destroyed. The liver is by no means the only organ from which transaminases can be liberated when cell damage occurs. Abnormal transaminase levels must, therefore, always be considered in conjunction with other tests and with the clinical picture. When necrotic heart or skeletal muscle cells are the source of the serum transaminases, the level of the serum glutamicpyruvic transaminase is either normal or, if raised, well below that of the serum glutamicoxalacetic transaminase (Wroblewski and LaDue, 1956a). In liver damage both transaminase concentrations may rise, the serum glutamic-oxalacetic transaminase level is proportionately higher in acute conditions and lower in chronic damage (Wroblewski and LaDue, 1956b). The upper level of normal for both transaminases was taken as 40 units and with careful technique there was no variation in result. If duplicate estimations varied by more than 2 units the estimation was repeated. Extreme accuracy is only needed when the test is used for diagnosis of cardiac infarction and in this study duplicate estimations were not carried out after 1957.

\section{Clinical Analysis}

The analysis deals with 155 cirrhotic patients who have undergone 157 shunt operations between 1948 and October, 1959. The number of patients with cirrhosis of all types seen during this period was 347. Shunt operations were not done in more than half, either because there was no clinical indication, e.g., satisfactory response to medical treatment for 
ascites; cirrhosis discovered incidentally as disease associated with peptic ulcer, etc., or because they were too ill for operative treatment.

The subdivision into mild, moderate, and severe cirrhosis was arbitrary (Hunt, 1958), designed only to indicate roughly how the patients would be expected to react to any major operation; the mild at little and the moderate at reasonable risk, but the severe at considerable danger. Many physicians have cooperated with us at every point and we are most grateful to them for their help. All modern methods of medical treatment have been used as they have been introduced.

\section{RESULTS}

The results have been assessed as follows.

"Success" means that a patient has been restored to normal life and work, without haemorrhage or ascites, indicating that portal hypertension has been relieved and liver function maintained. Death occurring from an intercurrent disease, e.g., carcinoma of bronchus, has not altered a previous decision to call the case a success, nor have mild and transient and controllable attacks of episodic stupor. The results recorded here are appreciably better than those recorded in 1958 .

"Late post-operative deterioration" infers that the patient recovered from the operation and later went downhill. Many have succumbed from liver failure, often preceded by attacks of episodic stupor. However, a proportion of the patients falling into this group were able to lead normal, useful, and happy lives for months or even years, in spite of diminishing liver function. In this sense the operation itself must be considered successful in that the risk of haemorrhage was removed and ascites eliminated, though the operation had done nothing to delay the progression of the liver disease. The terminal deterioration in such cases is often precipitated by an intercurrent disease, or some intervention of no greater severity than dental extractions. In this group are included those whose shunts have thrombosed and in whom the operation was of no benefit, though the patients survived.

"Post-operative deaths" include all patients dying in hospital following operations and those dying of complications resulting from the operation itself. We recognize that the death rate among early cases, certainly before 1951, was higher than it is today because our methods of selection were faulty and the patients were not, according to our present standards, always fully prepared, nor was our operative technique so practised, nor were our methods of pre- and post-operative treatment so effective or precise.

Lastly, it must be remembered that a pat:ent with cirrhosis has not the ability to resist intercurrent disease that is possessed by those fortunate enough to have normal livers, especially if an active parenchymatous lesion is present. Operations are undertaken, and indeed their whole lives are lived at added risk.

\section{Method of Tabulating Results}

The following graphs, Figs. 1, 2a and $b, 3 a$ and $b$, show the serum albumin and pseudocholinesterase levels for the three groups of patients: the mild, the moderate, and the severe cirrhotics. The graphs were compiled from figures obtained by taking the average reading for each patient over a particular period, the pre-operative phase and during each year post-operatively. Excluded from the analysis were the especially low readings encountered after a severe haemorrhage or during the immediate postoperative phase. The number of cases from which the readings were obtained is recorded for each period (the figure below the lower limit of variation for each period), but it may be short of the number of patients followed up through that particular year because the graphs do not take account of the patients who work abroad and can only attend for follow-up at irregular intervals. Each figure may, however, represent the average of a considerable number of readings, especially during the first post . operative year when the liver function tests may be repeated at two- or three-monthly intervals. The average, the highest, and the lowest figures are recorded.

The graphs were originally compiled as scattergrams, but the mass of detail was such as to confuse the issue. In the simplified form in which they are presented, clarity has been obtained at the expense of emphasizing the variations above and below the normal for each group of patients. It is, however, only the exceptional case that falls far wide of the average. Occasionally, in the assessment of a patient, the translation of a reading from "a statistic" into a figure of unique importance for an individual becomes a matter of some difficulty, but it must be borne in mind that it is only in this article that we are emphasizing certain biochemical tests. Much other material evidence-clinical,biochemical, and radiological-is normally considered in deciding the course to be adopted for a particular patient.

The Mild Cirrhotics.-These are patients who do not show any material evidence of hepatic disease pre-operatively and whose liver functions are within normal limits. Twenty-four out of 27 could be genuinely classified as long-term successes, giving a total of $89 \%$ success. There was no post-operative death. The liver function has remained normal during the whole period during which these patients have been 


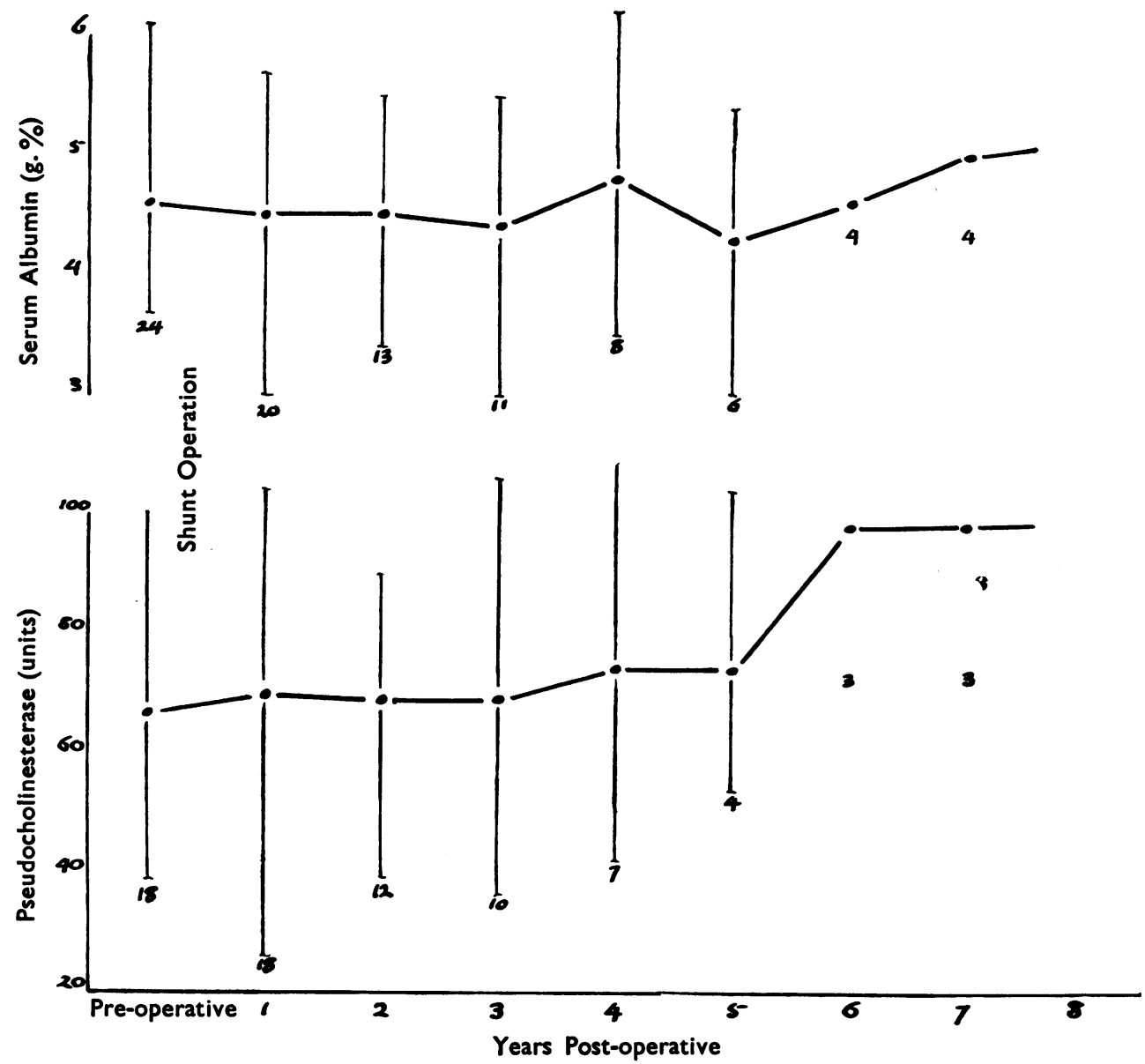

FIG. 1.-Serum albumin and pseudocholinesterase levels in 24 mild cirrhotics who have undergone successful operation for porta-caval or lieno-renal anastomosis. There is a tendency for the liver function, which is good pre-operatively, to improve post-operatively over the years.

under observation (up to more than 10 years), the serum albumin at 4.5 g. $\%$ (4.6 g. $\%$ pre-operatively) and the pseudocholinesterase at 70 (Warburg) units (67 units pre-operatively) (Fig. 1).

The Moderate Cirrhotics.-These are patients who show evidence of liver disease clinically or on biochemical assessment, but whose condition is not in the first place so severe as to preclude a shunt operation. They numbered $88 ; 56$ have been successfully operated on and 22 have deteriorated over the years, but in 17 of these the porta-caval anastomosis benefited them, giving a total of $83 \%$ materially improved by the anastomosis. Postoperative deaths numbered $10(11 \%)$.

In the successful group (Fig. 2a) the serum albumin pre-operatively was $4.4 \mathrm{~g} . \%$ and during the first four to five years since operation has not, on average, dropped below $4 \cdot 1 \mathrm{~g} . \%$. The pseudocholinesterase, 60 units pre-operatively, has fluctuated during the four years between 58 and 64 units. The picture is much the same as for mild cirrhosis, though on a slightly lower level.

In those who deteriorated (Fig. 2b) the serum albumin pre-operatively was $4.3 \mathrm{~g} . \%$, almost identical with the former group, but it did, on average, sink to $3.5 \mathrm{~g} . \%$ before terminal deterioration. The pseudocholinesterase, 49 units preoperatively (11 units less than the successful group), fell off rapidly to 32 units.

Of the 10 who died post-operatively, the preoperative serum albumin was $4.1 \mathrm{~g} . \%$ and the pseudocholinesterase 70 units. Death, on the whole, was not related to any deficiency in liver function, but to a number of other matters, of which technical difficulties played a considerable part. 
Fig. 2.-Serum albumin and pseudocholinesterase levels in moderately cirrhotic patients: A, 56 patients who have had a successful shunt operation and in whom the liver function is maintained; $B, 22$ patients in whom there has been deterioration of liver function postoperatively. (The pre-operative pseudocholinesterase level in $B$ is disproportionately low.)

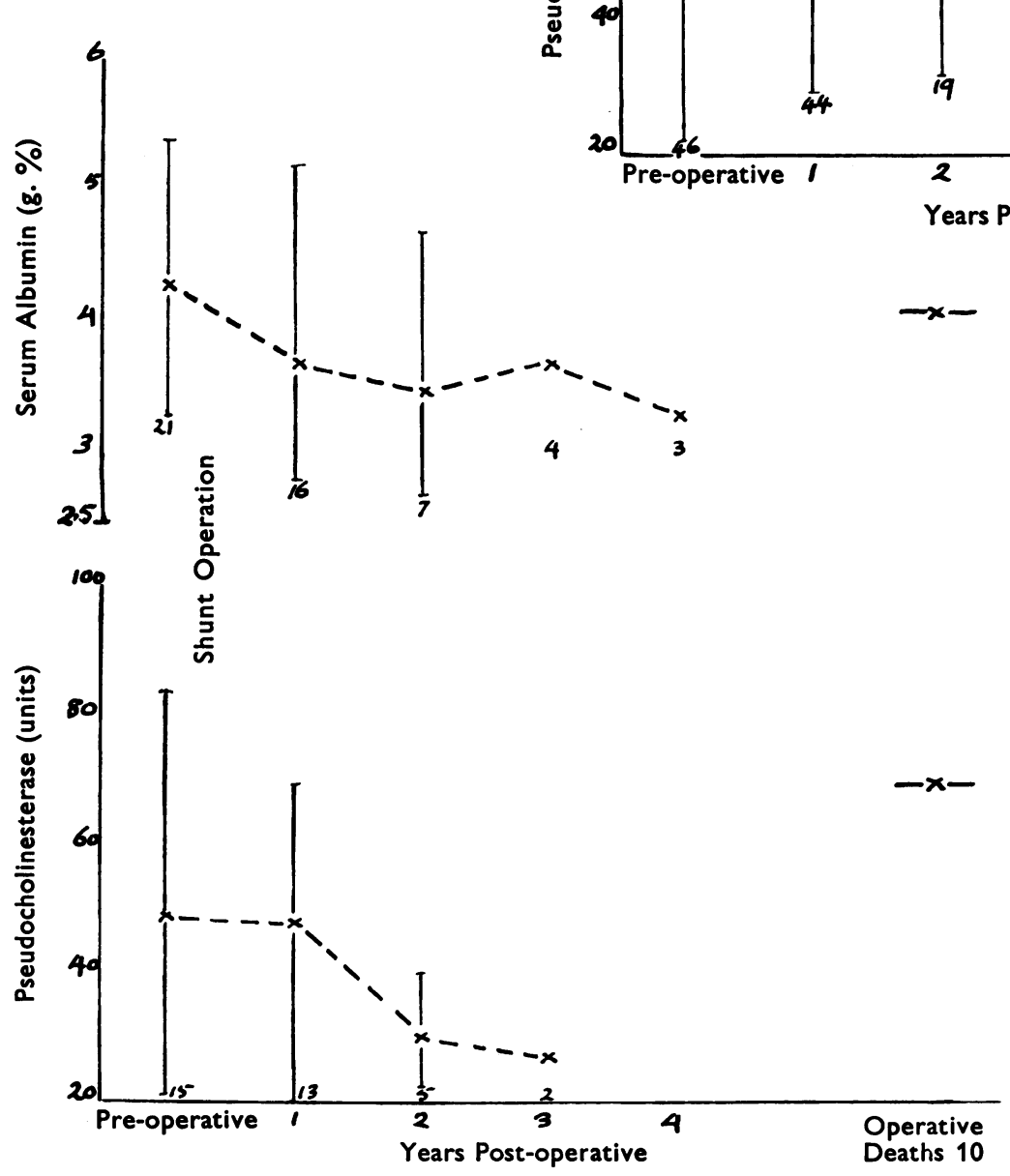


It will be noted that although the pre-operative level of serum albumin in moderate cirrhosis is the same in the groups that do well and in those that do badly, the average level of pseudocholinesterase is appreciably lower in the second group. A glance at the graphs will make it obvious that the assessment of an individual patient is a difficult matter because of the wide variations in the level of pseudocholinesterase. However, a low level or a change for the worse is undoubtedly a pointer towards ultimate liver failure. This is shown well during the second year of follow-up in the patients who deteriorated. At a time when the average serum albumin dropped only 0.2 g. $\%$ (from 3.7 to $3.5 \mathrm{~g} . \%$ ), the pseudocholinesterase fell from 48 to 32 units.

The Advanced Cirrhotics.-These are the patients who, when first seen, were considered too ill to tolerate a shunt operation on account of the advanced state of their liver disease, sometimes related to serious illness affecting other vital organs. Most patients in this group never improved sufficiently for a definitive operation to be undertaken. With intensive medical treatment lasting at least three and sometimes for 10 months, 40 attained a state in which a shunt operation was deemed justifiable. The serum albumin in nine patients rose during the pre-operative phase from an average of $2.5 \mathrm{~g} . \%$ to an average of $3.8 \mathrm{~g} . \%$.

Twenty-one (52.5\%) did well (Fig. 3a). Over the first three post-operative years the serum albumin has fluctuated between 3.8 and $4 \mathrm{~g} . \%$ and the pseudocholinesterase, 46 units pre-operatively, has improved to between 56 and 58 units during the same period.

It is only coincidence that the level of 46 units in this group of advanced cirrhotics who do well is identical with the level in the group of moderate cirrhotics who do badly, illustrating the point that most of the biochemical tests of liver function must be evaluated in relation to the clinical state of the individual. The serum albumin provides the absolute standard of comparison; the pseudocholinesterase indicates a trend.

The findings in this group suggest that if a patient responds well to medical treatment pre-operatively, the prognosis is good. Some of the patients who have done best of all have required many months of intensive medical treatment before reaching a state at which the risk of operation has been justified. The figures in this group prove beyond shadow of doubt that liver function may actually improve following shunt operations and that prolonged and persistent pre-operative treatment in an apparently hopeless case sometimes pays excellent dividends.

Seven advanced cirrhotics have deteriorated post- operatively (Fig. 3b), but four were temporarily improved, so that one can say that altogether in 25 (or $62 \%$ ) a shunt operation has been of great value. Pre-operatively the average serum albumin level was $3.8 \mathrm{~g} . \%$ (identical with the successful group) and the pseudocholinesterase 36 units (10 units less, be it noted again, than in the successful group).

In the advanced cirrhotics who died postoperatively (Fig. 3b), the serum albumin level averaged 3.5 g. \% pre-operatively and the pseudocholinesterase 36 units. They number 12 and constitute $30 \%$ of this very ill group. However, many of these patients had not been considered fit for operation by other surgeons interested in portal hypertension and most had only attained a reasonable state after prolonged and intensive medical treatment. Many would never have been included in other surgical series so the unduly high operative mortality in this group must be viewed in correct perspective. At the expense of a $30 \%$ operative mortality, much has been gained for those patients who have survived.

All Groups.-Fig. 4 has been drawn to emphasize the significance of the results of Figs. 1, 2, and 3. The proportionately lower placement by 10 or 11 units of the pseudocholinesterase levels preoperatively in those patients who ultimately die becomes more evident. The serum albumin in each group (related to the degree of cirrhosis) is by contrast the same.

The advanced group contains most of the ascitic patients. Usually they have also had haemorrhages and some were jaundiced. It is often said that ascites and jaundice are contraindications to shunt operations, but this is not necessarily so, provided the liver function recovers reasonably well, as indicated by the serum albumin rising to above $3.2 \mathrm{~g} . \%$ and pseudocholinesterase levels improving.

Altogether 35 patients have had gross ascites at the time of their portal-systemic venous anastomosis (reported by Knipe, 1959). All who survived operation have been temporarily or permanently relieved of ascites and 25 have been enabled to return to full work or normal life $(71 \%)$. Clinically the prospects of a patient with intractable ascites, which has failed to respond to medical treatment, are bad; but we think that we can, by these biochemical tests, select those patients who may be expected to benefit from operative treatment.

\section{TRANSAMINASES}

So far in this analysis we have dealt with the two tests which we consider to be particularly valuable in assessing prognosis. This does not infer that we 
Fig. 3-Serum albumin and pseudocholinesterase levels in patients with advanced cirrhosis. $A$, in 21 patients before and after successful shunt operation, with liver function well maintained; $B$, in seven patients when there has been ultimate liver failure. Again, the preoperative pseudocholinesterase level in $B$ is disproportionately low.
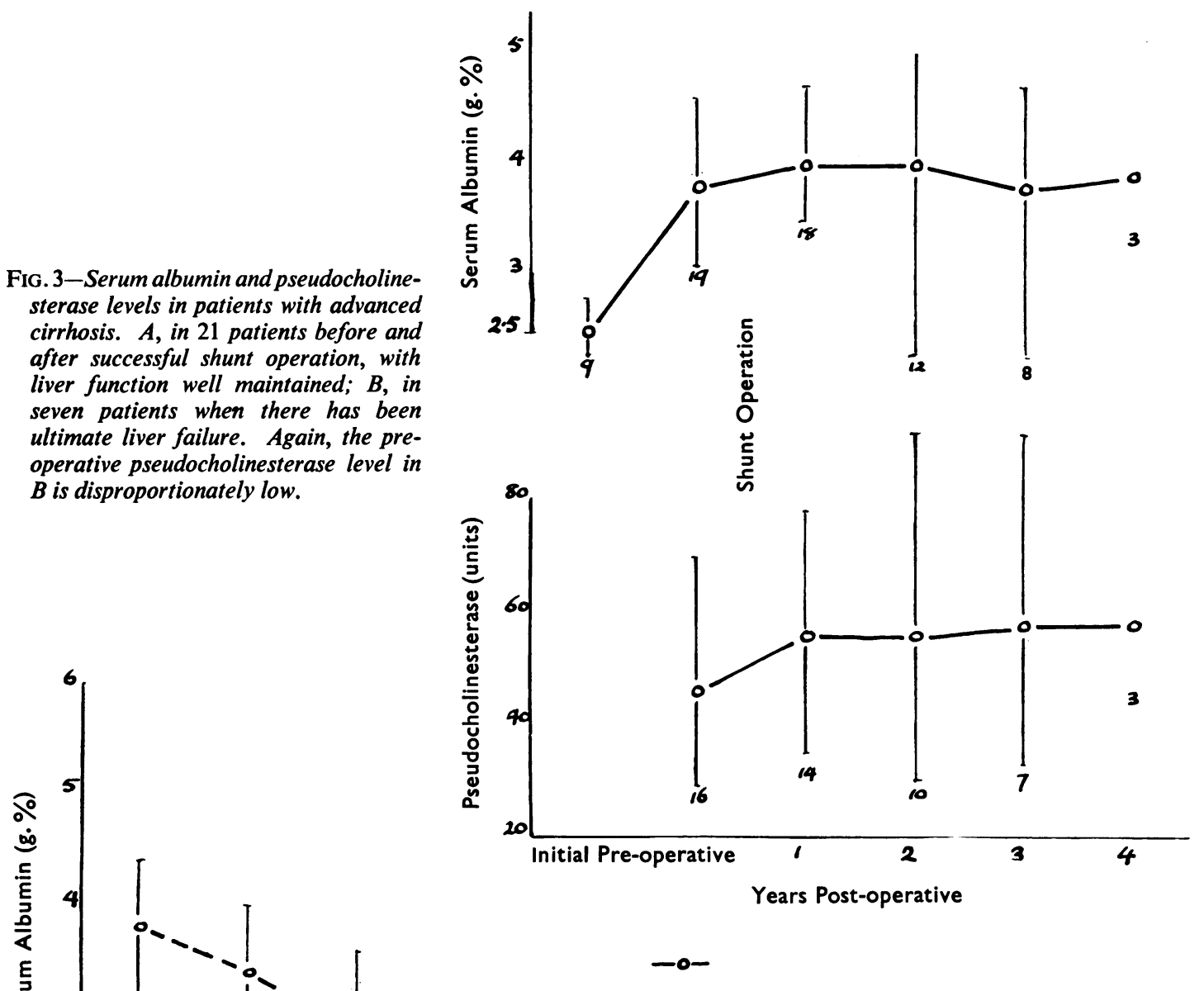


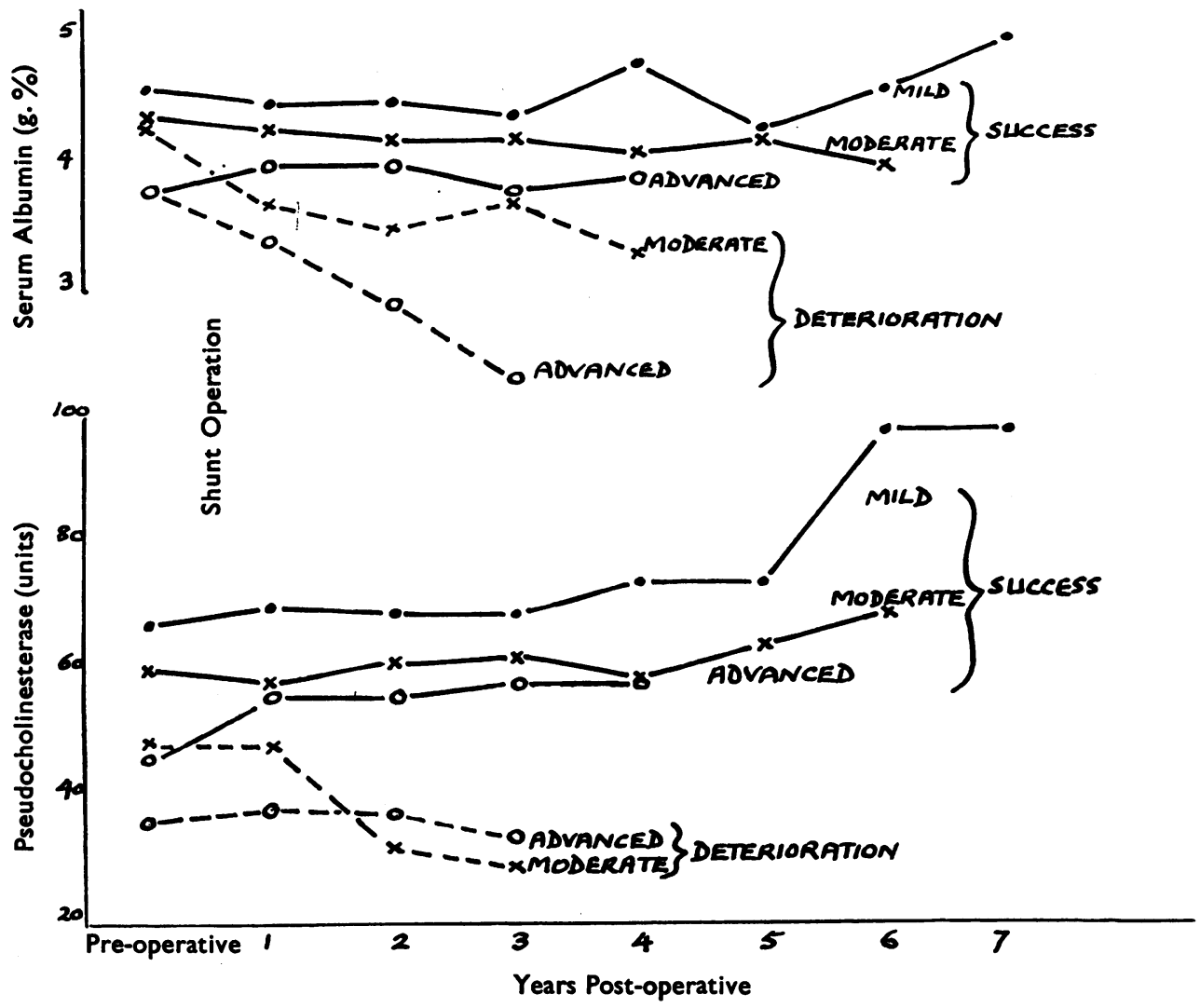

FIG. 4.-Composite graph emphasizing the prognostic value of pseudocholinesterase in the pre-operative assessment of patients with moderate and advanced cirrhosis.

have restricted our biochemical investigations of liver disease to these two tests alone. We use many others when we feel they are indicated. Recently we have been investigating the serum glutamic-oxalacetic and glutamic-pyruvic transaminases in the hope that they would indicate the continued activity of a pathological process within the liver and thereby warn us of an unfavourable circumstance. So far, however, we have not been able to come to a definite conclusion about their value, because the levels have varied greatly and a raised transaminase level does not necessarily indicate an overall incapacity of the liver to carry out reasonable function; nor is a "normal" level or a fall in the transaminase level invariably an indication that no destruction of liver cells is going on, or that the pathological process is regressing. So many of the cells may have been destroyed already that the remainder, though injured, do not contribute much enzyme to the serum. In such patients a fall in the transaminase levels may be a bad rather than a good sign, especially if associated with a fall in the pseudo- cholinesterase level. Transaminase activity should therefore, be considered in conjunction with other tests.

The average levels have been as follows:

\begin{tabular}{|c|c|c|c|}
\hline & & Pre-operative & Post-operative \\
\hline $\begin{array}{l}\text { Mild cirrhosis } \\
\text { (5) } \\
\text { Moderate } \\
\text { (good results, 20) } \\
\text { Moderate } \\
\text { (poor results, 6) } \\
\text { Severe } \\
\text { (good results, 8) }\end{array}$ & $\begin{array}{l}\text { S.G.-O.T. } \\
\text { S.G.P.T. } \\
\text { S.G.-O.T. } \\
\text { S.G.-P.T. } \\
\text { S.G.-O.T. } \\
\text { S.G.-P.T. } \\
\text { S.G.O.T. } \\
\text { S.G.-P.T. }\end{array}$ & $\begin{array}{l}\left.\begin{array}{l}31 \\
28 \\
96 \cdot 5 \\
63 \\
35 \\
24 \\
80 \cdot 5 \\
63 \cdot 5\end{array}\right\} \text { Normal } \\
\text { Elevated }\end{array}$ & $\begin{array}{l}30 \\
21 \\
67 \\
43 \\
66 \\
37 \\
82 \cdot 5 \\
38\end{array}$ \\
\hline
\end{tabular}

We may be able in time to detect the likelihood of improvement or deterioration as judged by the degree of activity. Two successful cases illustrate this hope.

The first, a boy with congenital cystic disease of the pancreas, cirrhosis, and bronchiectasis, showed the following fluctuations in transaminase: S.G.-O.T. pre-operatively 160 , improving to 55 with medical treatment, deteriorating to 135 postoperatively, and then improving to 58 units; S.G.-P.T. pre-operatively 110 , improving to 58 , 
deteriorating to 80 post-operatively, and then improving to 17 units. The boy is doing well 19 months after operation.

The second, a man with gross ascites and haemorrhage, who has done extraordinarily well, showed the following improvement during three months' pre-operative treatment: S.G.-O.T. 30 to 14 units, S.G.-P.T. 48 to 22 units.

\section{ConClusion}

In studying the problem of liver disease from a surgical point of view, there is need for a test (or group of tests) which will enable us to distinguish clearly in the individual pre-operatively the patient who will not only tolerate a shunt operation but also not deteriorate later. That no such test has as yet been available may be a blessing in disguise, in that hope has not yet to be withheld from the patient who will ultimately deteriorate. A shunt in such circumstances may bring much relief and even great happiness, even though life may not be prolonged beyond a few years, but these are lived without the threat of devastating haemorrhage and the discomfort of incapacitating ascites. The serum pseudocholinesterase, we believe, gives us prior information of value and follow-up estimations show what the clinical trend will be in advance of other findings. It also responds much more rapidly to day-to-day changes in the state of the liver. Taken in conjunction with the serum albumin, its estimation is, therefore, of great value. Further, it must not be forgotten that a level of less than 55 units is almost certainly diagnostic of liver disease, whatever the other tests show. We are led to the emphatic conclusion that, in our experience, the serum albumin and serum pseudocholinesterase levels are the two most important estimations in helping to assess the prognosis of a case of cirrhosis before operation and during the follow-up period. The whole subject, however, of portal hypertension is of such complexity that the biochemical laboratory can be no more than ancillary and we fully recognize that clinical appraisal is at least as important as any or all the liver function tests.

\section{SUMMARY}

One hundred and fifty-five patients with cirrhosis, in whom shunt operations have been done, have been analysed in order to relate the clinical outcome on the one hand to the extent of the cirrhosis on the other, the liver function being assessed by estimation of the serum albumin and pseudocholinesterase levels.

The serum albumin gives, on the whole, an accurate assessment of the overall liver function. We confirm that no shunt operation should be undertaken in a patient whose serum albumin is less than $3.2 \mathrm{~g} . \%$.

The pseudocholinesterase level is of supplementary value and, although the range of normal values is wide, it appears that $30-35$ units is a critical level. Low readings are of value diagnostically, but the most important assistance to be derived from the pseudocholinesterase is that it gives an indication of whether or not the patient will undergo late deterioration of liver function after a shunt operation. A material falling off in the pseudocholinesterase level is seen before the serum albumin alters significantly. Day-to-day changes are sometimes reflected by this test.

An assessment of the transaminases is not yet possible, but it appears that they will have to be evaluated in conjunction with the other tests of liver function so that the amount of enzyme liberated is related approximately to the total quantity of functional liver substance present.

The analysis has shown conclusively that liver function may not only be maintained for years following a shunt operation but that it may actually improve. The methods of assessment described in this article go part of the way to indicating, before operation, what the ultimate result will be. A shunt operation has proved successful or worthwhile in $87 \%$ of the mild cirrhotics and in $83 \%$ of the moderate cirrhotics. Even in the advanced cirrhotics, a group of patients normally precluded from this operation, a proportion become suitable for a shunt and of these $62 \%$ have done well.

We should like to thank Mr. H. F. Weston who supervised the estimation of nearly all albumin values, and Mrs. E. Silk, Mrs. H. Kay, Miss E. Ryan, and Miss V. Patston who as research assistants carried out estimations of pseudocholinesterase. We should like to thank the Medical Research Council who provided a research assistant from 1951 to 1954 and the Governors of St. Bartholomew's Hospital who continued this financial support from 1954 to 1959.

\section{REFERENCES}

Blakemore, A. H. (1949). Surg. Gynec. Obstet., 89, 357-359.

Harris, H., Whittaker, M., Lehmann, H., and Silk, E. (1960). Acta genet. (Basel), 10, 1-16.

Harrison, G. A. (1937). Chemical Methods in Clinical Medicine, 2nd ed. Churchill, London.

Hawk, P. B., Oser, B. L., and Summerson, W. H. (1947). Practical Physiological Chemistry, 12th ed. Blakiston, Philadelphia.

Hunt, A. H. (1958). Portal Hypertension. Livingstone, Edinburgh.

Jones, M. S., and Tod, H. (1935). Biochem. J., 29, 2242-2245.

Kalow, W., and Genest, K. (1957). Canad. J. Biochem., 35, 339-346.

T. and Gunn, D. R. (1959). Ann. hum. Genet., 23, 239-250.

King, J. (1958), J. med. lab. Techn., 15, 17-22.

Knipe, P. (1959). Proc. Roy, Soc. Med, 52 249-252.

Lehmann, H., Patston, V., and Ryan, E. (1958). J. clin. Path., 11, 554-553.

and Ryan, E. (1956). Lancet, 2, 124.

- and Ryan, E. (1956). Laince

McArdle, B. (1940). Quart. J. Med., n.s., 9, 107-127.

Reitman, S., and Frankel, S. (1957). Amer. J. clin. Path., 28, 56-63.

Wroblewski, F., and LaDue, J. S. (1956a). Ann. intern. Med., 45 801-811. 